\title{
A FAMILY WITH HYPOTRICHOSIS ASSOCIATED WITH CONGENITAL HYPOPLASIA OF THE THUMB
}

\author{
Akiyasu ChiBA* and Takayuki MiurA** \\ *Division of Orthopaedic Surgery, Gamagoori City Hospital, Gamagoori \\ **Department of Orthopaedic Surgery, Nagoya University \\ School of Medicine, Nagoya
}

\begin{abstract}
Summary A family with hypotrichosis associated with hypoplasia of the thumb was reported. A 3-year-old boy and his mother had remarkably fine and sparse scalp hair. Their thumbs and thenar muscles were markedly hypoplastic. Radiograph of the right hand of mother showed the thumb consists of three phalangeal bones. Teeth deformity, nail deformity, cataract and deformity of sweat function were not revealed.
\end{abstract}

\section{INTRODUCTION}

Hypotrichosis is a relatively common feature of many hereditary syndromes, usually in association with other ectodermal defects. There have been many reports of such syndromes with associated teeth deformities, defects of sweat function and craniofacial anomalies (hypohidrotic ectodermal dysplasia: Frias and Smith, 1968), atrophic dry skin and juvenile cataracts (Werner's syndrome: Werner, 1904), nail deformity, keratosis pilaris and erupted teeth at birth (pachyonychia congenita: Soderquist and Reed, 1968), nail hypoplasia and hyperpigmentation (Clouston-type ectodermal dysplasia: Smith, 1970), polydactyly and cardiac defects (Ellis-vanCreveld syndrome: Ellis and van Creveld, 1940) and prominent nose with Type-12 peripheral dysostosis (tricho-rhino-phalangeal syndrome: Giedeon, 1966).

However, to the best knowledge of the authors, there is no literature on congenital deformity of the thumb associated with sparse hair of the scalp. The purpose of this paper, then, is to describe a family with hypoplasia of the thumb associated with hypotrichosis.

\section{CASE REPORT}

Proband: M.M., a 3-year-old boy (III-2)

Present illness. Delivery was normal following an uneventful full-term pregnancy. The mother was 28 and the father 27 when the patient was born. The 


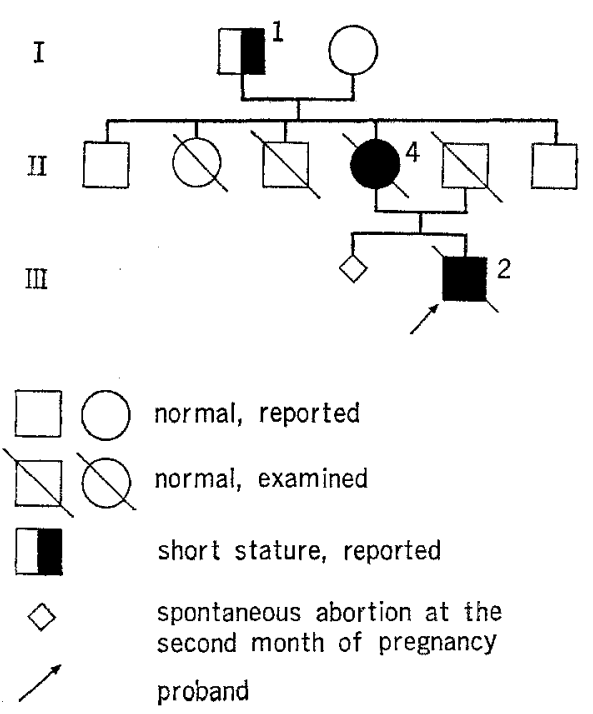

Fig. 1. Pedigree of the family.

infant showed functional disorder of both thumbs and hypotrichosis at birth. The mother has a previous history of a spontaneous abortion at the second month of gestation. There was no consanguinity in this family. The patient's maternal grandfather was reported to be of short stature. His father was alive and normal. The mother had the same symptoms as the proband. There was no sibling (Fig. 1).

Physical examination. Examination revealed physical and mental retardation. He walked at 20 months of age, babbled at 24 months (baba and mamma) and still cannot speak distinctly. His stature was markedly short, $78 \mathrm{~cm}$ as opposed to $98 \mathrm{~cm}$ for normal Japanese boys of the same age.

His scalp hair was remarkably fine and sparse. His eyebrows and eyelashes, however, were almost normal (Fig. 2). Dark skin pigmentation with pinhole-size white spots in it was present on the dorsal sides of both hands and forearms. The teeth and nails were normal. There was no disturbance of the heat-regulating function of the body.

The patient had strikingly hypoplastic thumbs. The thenar muscle was markedly hypoplastic in both hands and the thenar creases had disappeared. The metacarpophalangeal joints of both thumbs were unstable. As a result, he can not grip or pinch skillfully (Fig. 3).

The results of routine examinations of the blood and urine were within normal limits. The Wasserman reaction was negative. Chromosomal type was normal male, 46,XY. The electrocardiogram was normal.

$\mathrm{X}$-ray examination of the thumbs revealed marked hypoplasia of the first metacarpal and two phalangeal bones. The Turkish saddle deformity was not observed (Fig. 4). 
The mother: Y.M., a 31-year-old woman (II-4)

At birth, she manifested fingerlike hypoplastic thumbs and sparse scalp hair. In adulthood, her height was $140 \mathrm{~cm}$ as opposed to $155 \mathrm{~cm}$ for normal Japanese

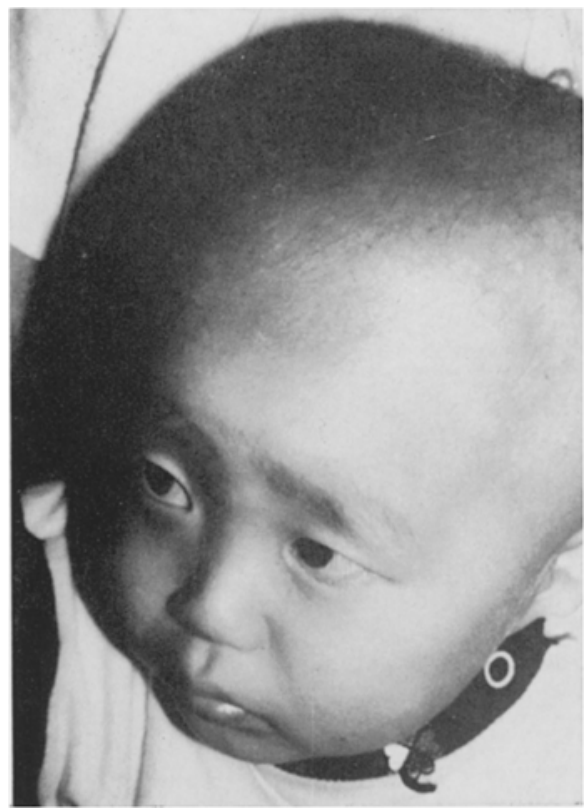

Fig. 2. External appearance of the head and face of the proband. His scalp hair is remarkably fine and sparse.

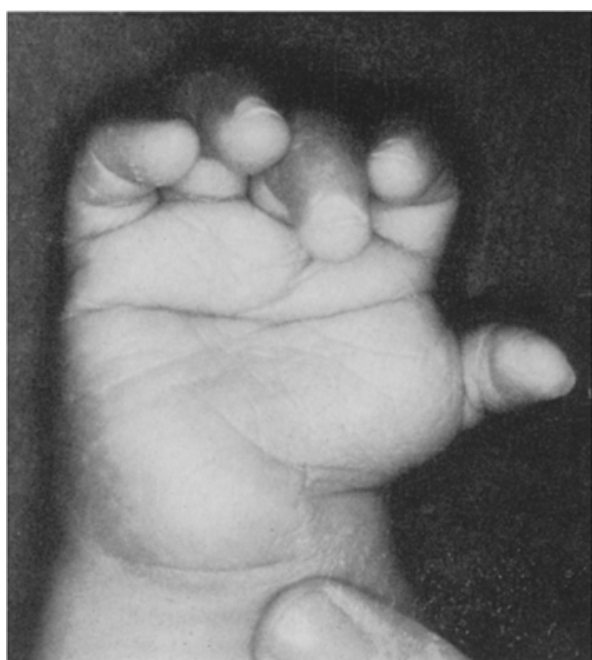

Fig. 3. The right hand of the proband. Thenar muscle and thumb are markedly hypoplastic. Pinhole-size white spots are present on the dorsal side of fingers.

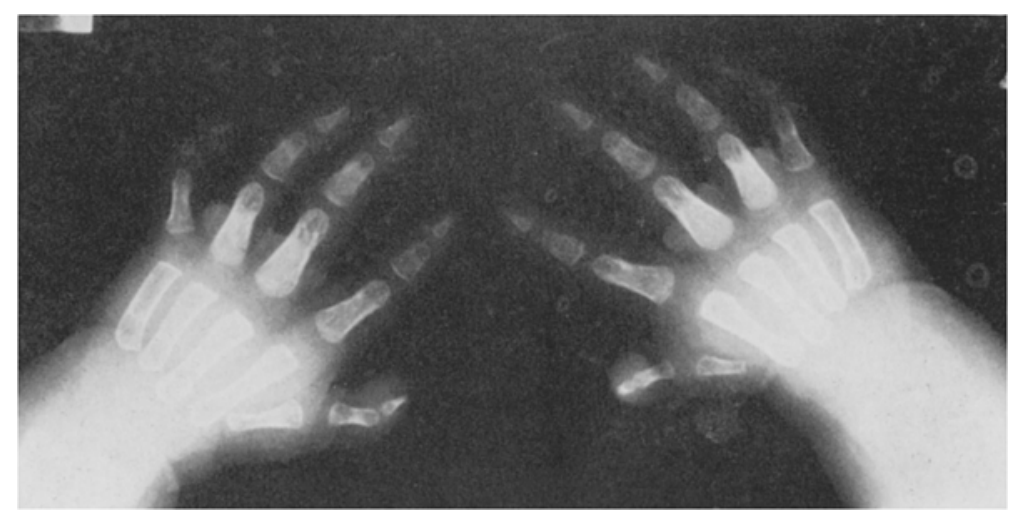

Fig. 4. Radiograph of both hands of the proband. 


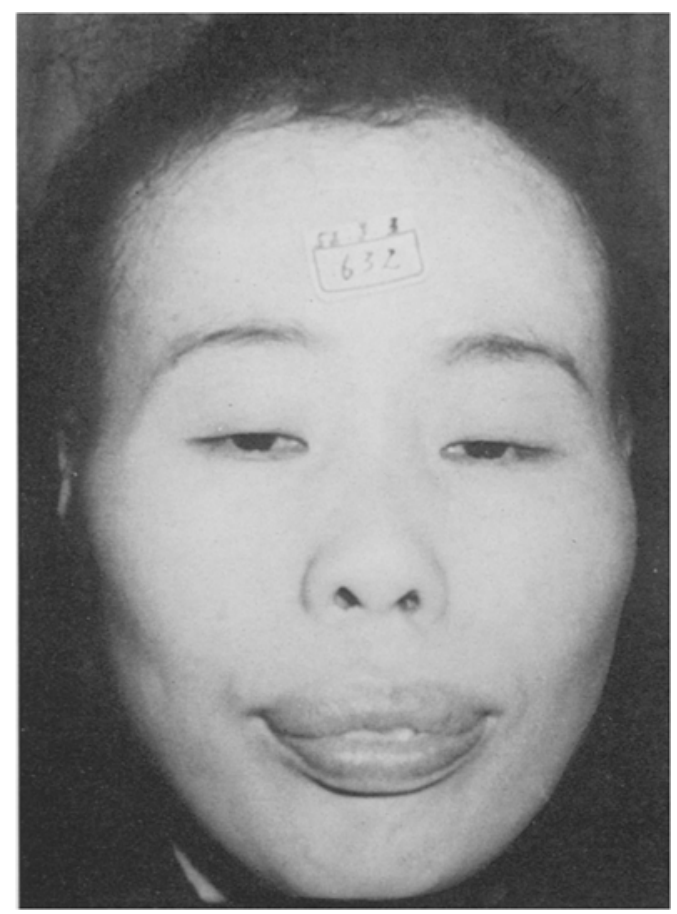

Fig. 5. External appearance of the headinand face of mother. Sparsity of hair and slightly prominent lip are showed.

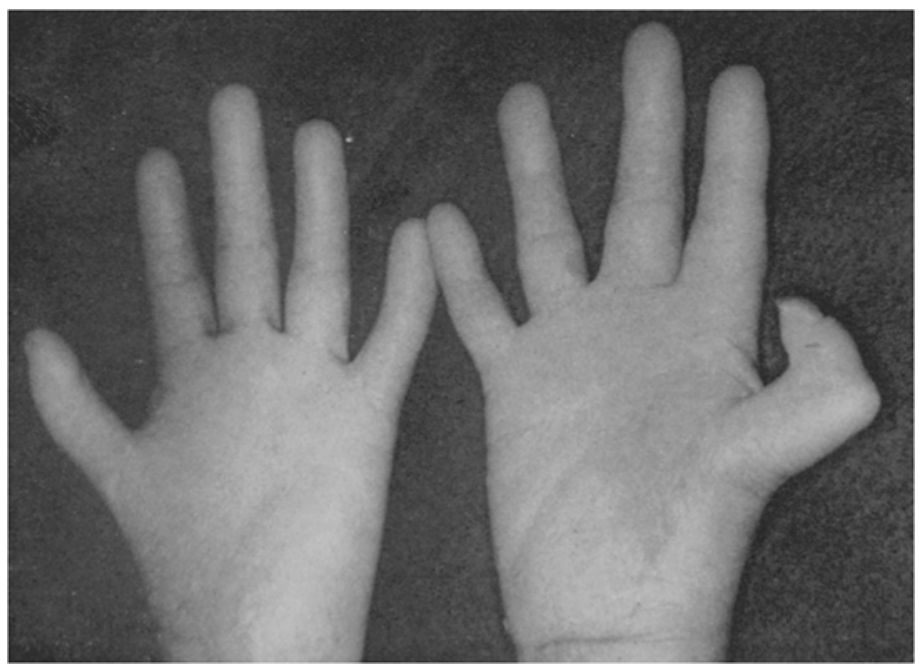

Fig. 6. The both hands of mother. Thenar muscle are markedly hypoplastic and both first interdigital spaces are narrow. The right thumb shows flexion contracture of IP joint and the distal phalanx is deviated ulnar side. 


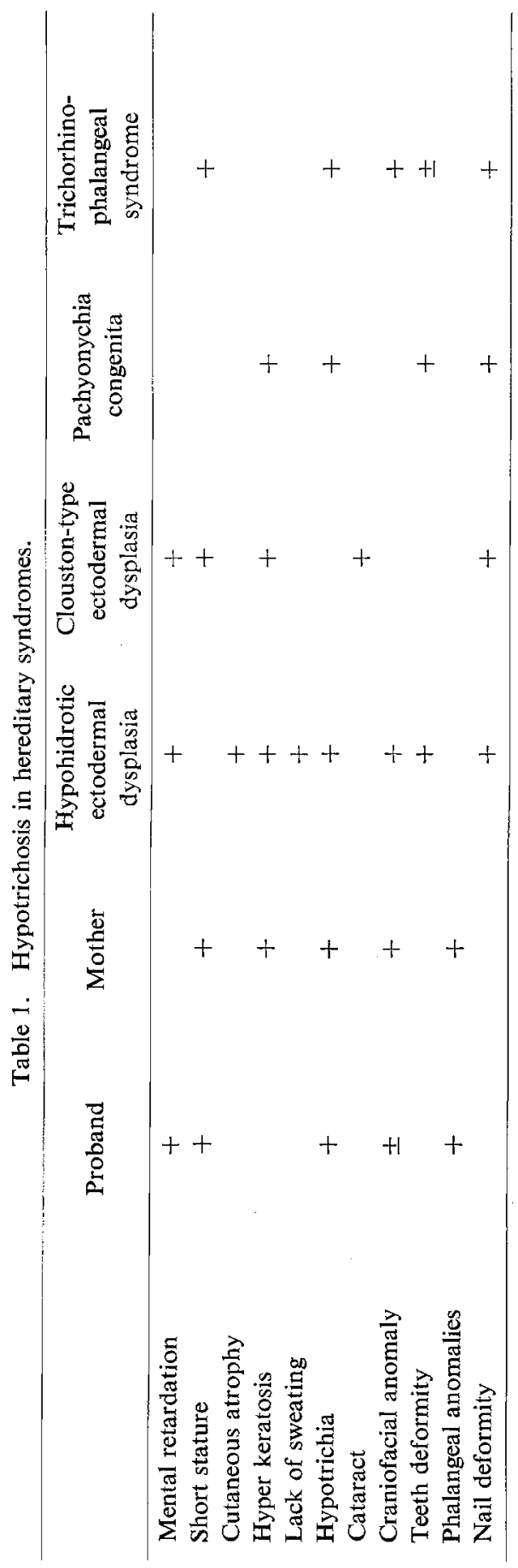




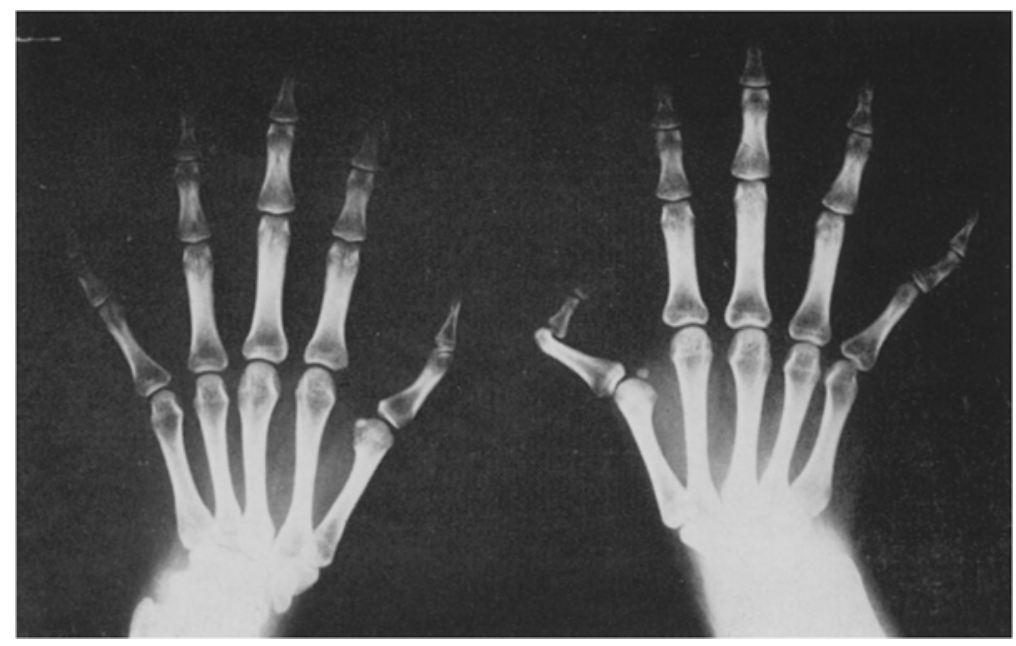

Fig. 7. Radiograph of the both hands of mother. This shows the right thumb consists of three phalangeal bones.

females. Her scalp hair was markedly fine and sparse. The eyebrows, eyelashes and axillary hair, however, were normal. Her lips were slightly prominent (Fig. 5).

The thenar muscle was markedly hypoplastic, and the thenar crease had disappeared in both hands. The first interdigital space was narrow. The right thumb showed flexion contracture of the interphalangeal joint. The distal phalanx was deviated ulnarside at the interphalangeal joint. Dark skin pigmentation with pinhole-size white spots in it was present on both hands and forearms. Mild hyperkeratosis of the palms and the soles were noted (Fig. 6).

$\mathrm{X}$-ray examination of the right thumb showed hypoplasia of the first metacarpal and three phalangeal bones. X-ray examination of the left thumb showed hypoplasia of the first metacarpal and two phalangeal bones (Fig. 7).

\section{COMMENTS}

The mode of transmission in the present case seems to be autosomal dominant. There are many syndromes associated with hypotrichosis and other ectodermal dysplasia, as shown in Table 1. None of them, however, is consistent with the symptoms noted in this family. Thus, to the best of our nowledge, the combination of anomalies presented in this paper represent a new syndrome of ectodermal dysplasia.

\section{REFERENCES}

Ellis, R. W. B. and van Creveld, S. 1940. A syndrome characterized by ectodermal dysplasia, 
polydactyly, chondro-dysplasia and congenital morbus cordis. Report of three cases. Arch. Dis. Child. 15: 65-84.

Frias, J. L. and Smith, D. W. 1968. Diminished sweat pores in hypohidrotic ectodermal dysplasia: A new method for assessment. J. Pediatr. 72: 606-610.

Giedeon, A. 1966. Das Tricho-rhino-phalangeale Syndrom. Helv. Pediat. Acta 21: 475-482.

Smith, D. W. 1970. Recognizable Patterns of Human Malformation, Saunders, Philadelphia, pp. 182.

Soderquist, N. A. and Reed, W. B. 1968. Pachyonychia congenita with epidermal cysts and other congenital dyskeratoses. Arch. Dermat. 97: 31-33.

Werner, O. 1904. Über Katarakta in Verbindung mit Sklerodermie (Doctoral dissertation, Kiel University). Schmidt und Klaunig, Kiel. 